\title{
STUDIES ON LIGHT SCATTERING SPECTRUM OF MICROBIAL CELLS IN SUSPENSION
}

\author{
SHOZO KOGA and TERUYUKI FUJITA \\ The Institute of Applied Microbiology, University of Tokyo \\ Received for publication, May 13, 1961
}

\section{INTRODUCTION}

As described in a previous paper (1), spherical microbial cells in suspension could approximately be regarded as optically homogeneous scatterers with total cross sections expressed by Rayleigh-Gans' or Hart-Montroll's formulae. In the present paper, it is shown that the wave-length dependence of turbidity can serve as "light scattering spectrum" for obtaining biological information on cellular level. In order to carry out the light scattering analysis of this kind with respect to microbial suspensions, a commercially available spectrophotometer could conveniently be used as shown later instead of the one mentioned in the preceding paper. General procedure of the analysis will be dealt with and the outcome will be applied to microbiological problems with special regard to Saccharomyces cerevisiae.

\section{THEORY AND APPARATUS}

To facilitate the discussion, the basic equations described in the preceding paper(1) for light scattering of spherical cells in suspension will be cited as follows with a graphical representation in Fig. 1:

$$
\begin{aligned}
& \sigma_{s} / \pi a^{2}=(m-1)^{2} \phi(x), \\
& \phi=(5 / 2)+2 x^{2}-(\sin 4 x / 4 x)-\left(7 / 16 x^{2}\right)(1-\cos 4 x) \\
&+\left\{\left(1 / 2 x^{2}\right)-2\right\}\left\{0.577+\operatorname{los} 4 x-C_{i}(4 x)\right\}, \\
& C_{i}(x)=-\int_{x}^{\infty}(\cos u / u) d u . \\
& \sigma_{s} / \pi a^{2}=\left\{\pi x^{2}(m-1)^{2} / 2 m\right\}\left\{\left(m^{4}+6 m^{2}+1\right) \Delta_{1}-2\left(m^{2}+1\right) x^{-2} \Delta_{2}+x^{-4} \Delta_{3}\right\}, \\
& \Delta_{j}=I_{j}[x(m+1)]+I_{j}[x(m-1)], \\
& I_{1}(2 x)=(1 / 2 \pi)-(1 / 8 \pi)\left\{J_{1 / 2}^{1}(2 x)+_{3 / 2}^{2}(2 x)\right\}, \\
&\left.I_{2}(2 x)=(1 / \pi)\{\sin 4 x / 2 x)-\left(\sin ^{2} 2 x / 4 x^{2}\right)-1+\varphi(4 x)\right\}, \\
&\left.\left.I_{3}(2 x)=(1 / \pi)\right\} 2 x^{2}+x \sin 4 x-(5 / 2) \sin ^{2} 2 x+\varphi(4 x)\right\}, \\
& \varphi(z)=\int_{0}^{z}(1-\cos y) d y / y . \\
& a: \text { radius of spherical cells }
\end{aligned}
$$


$n_{0}$ : index of refraction of suspending medium

$n_{1}$ : index of refraction of suspended cells

$\lambda_{0}$ : wavelength of light in the suspending medium

$\sigma_{s}$ : cross section of light scattering

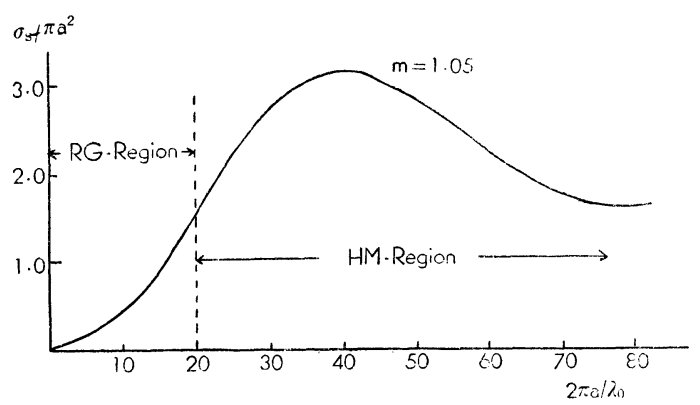

Fig. 1. Light scattering cross section for spherical scatters (relative index of refraction: 1.05).

Since index of refraction $m$ is of values close to unity referred to the medium in all live cells, corresponding first approximate forms to equs. (1) and (2) are, respectively:

$$
\begin{aligned}
\sigma_{s} / \pi a^{2} & =(m-1)^{2} \cdot 2 x^{2}=(1 / 2) \rho^{2} \quad(1<x<20), \\
\sigma_{s} / \pi a^{2} & =2-4 \rho^{-1} \sin \rho+4 \rho^{-2}(1-\cos \rho) \quad(x \geq 20), \\
\rho & =2 x(m-1) .
\end{aligned}
$$

In Fig. 1, the ratio of cross section of light scattering to geometrical cross area is drawn for ratio parameter $x \equiv\left(2 \pi \alpha / \lambda_{0}\right)$ by using equ. (1) over the range $x<20$ and equ. (2) over the range $x \geq 20$. As numerically seen from equ. (4), the curve in this figure shows a maximum at wavelength $\lambda_{\max }$ where

$$
4 \pi a(m-1) / \lambda_{\max }=2.08 .
$$

For obtaining accurate light scattering spectrum, transmission measurements at various wavelengths must be made under geometrical condition of narrow beam as mentioned, otherwise observed turbidity may become low owing to the light scattered into a small angle viewed off the direction of incident beam. With due consideration on this geometrical requirements, a single beam apparatus of indicating type was constructed as described in the preceding paper for studies on absolute values of light scattering cross section shown by spherical cells in suspensions. Since commercial spectrophotometers used in chemical studies are mostly designed for measurements of absorption in solutions or for purposes of practical turbidimetry in limited cases, they do not always meet the requirements for absolute turbidimetry. Therefore, a preliminary test was made on one of those spectrophotometers, Beckman Model DK-2, to see whether or not the type could be used for 
recording scattering spectra of microbiol suspensions. Since forward scattering is of higher intensity for cells of larger diameter, Saccharomyces cerevisiae was used as a specimen of large diameter, probably the largest among the microbes to be studied.

In Fig. 2 were drawn curves obtained by plotting apparent turbidity $\tau^{\prime}$ measured in absorbance scale in Beckman DK-2 against the population densities $N$ of the specimen used. In the range not exceeding 0.8 in absorbance scale, a linear relation holds between $\tau^{\prime}$ and $N$ for every wave length used. The linear region, which will be called hereafter "dilute region", corresponds to the range over which the effect of multiple scattering

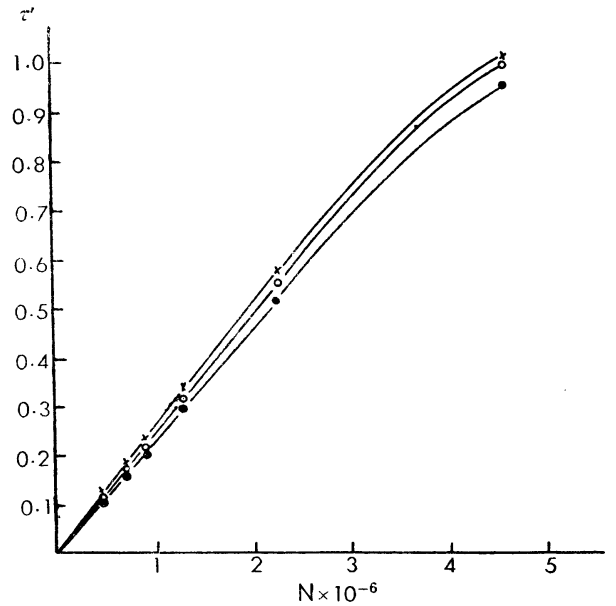

Fig. 2. Apparent turbidity vs. cell population.

$$
\times 504 \mathrm{~m}_{\mu} \quad \bigcirc 548 \mathrm{~m}_{\mu} \quad \text { - } 623 \mathrm{~m}_{\mu}
$$

can safely be disregarded so far as the data obtained by the use of this instrument is concerned. The absolute values of cross section of light scattering could, therefore, be found from inclinations of straight lines in Fig. 2. In Table 1 , the values $\left(\sigma_{s}^{2}\right)$ thus obtained are compared with those $\left(\sigma_{s}{ }^{1}\right)$ found by the use of standard narrow beam turbidimeter shown in a previous paper.

Table 1. Light scattering cross section at various wavelengths.

$\lambda(\mathrm{m} \mu)$
471
504
548
623

$\sigma s^{1}\left(\mathrm{~cm}^{2}\right) \times 10^{7}$
6.4
6.4
6.2
5.5

\begin{tabular}{c}
$\sigma_{s}^{2}\left(\mathrm{~cm}^{2}\right) \times 10^{7}$ \\
\hline 5.8 \\
5.7 \\
5.6 \\
5.2
\end{tabular}

Although, owing to the lack of exact narrow beam condition, values of $\sigma_{s}{ }^{2}$ were always somewhat smaller than the corresponding ones given in the row of $\sigma_{s}{ }^{1}$, the difference did not exceed $10 \%$ of the original values at most. Moreover, differences of this sort are smaller for microbes of smaller diameter in accordance with the theory on angular distribution of scattered light. Commercially available spectrophotometer, Beckman Model DK-2, was therefore used as a working apparatus throughout the present work in which approximate patterns of whole spectra were more concerned than accurate values of light scattering intensity at any definite wave length. 


\section{GENERAL PROCEDURES OF LIGHT SCATTERING ANALYSIS}

As theoretically expected of equs. (1) and (2), suspensions of cells of relatively large diameter might exhibit a maximum turbidity at a length in visible ranges. Examples of light scattering spectra are shown in Fig. 3 for spherical or quasi-spherical cells in dilute suspension. The observed average cells in dilute suspension. The observed average cell diameters $\langle D\rangle$ were $5.6 \mu, 4.1 \mu$ and $1.4 \mu$, respectively. From equ. (5), it can easily be proved that, at maximum wave length $\lambda_{\max }$, there exists an approximate relation

$$
\pi\langle D\rangle \Delta n / \lambda_{\max }=1.04
$$

where $\Delta n \equiv n_{1}-n_{0}$ and $\langle D\rangle$ indicates the average diameter of cells in suspension. If index increment $\Delta n$ did not noticeably vary in its magnitude among the species used, the value of $\lambda_{\max }$ for smaller cells might be expected to shift to the shorter wavelength side as shown in Fig. 3.

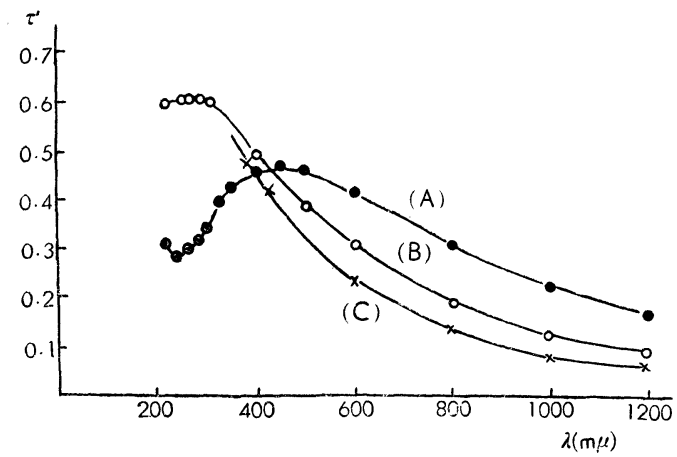
(A) Saccharomyces cerevisiae
(B) Rhodotorula glutinis
(C) Micrococcus cancidus

Fig. 3. Light scattering spectra.

In the following paragraphs, it will be shown that, by analyzing observed spectra of light scattering by means of the basic equations above described, one can evaluate in a perfectly spectrophotometrical manner the magnitude of cell diameter, cell index of refraction and population density of cellular suspensions.

\section{(a) Cellular Index of Refraction $n$ :}

A series of sample suspension was made by suspending the same population of a test microbe in the same volume of media with various indicies of refraction $\left(n_{0}\right)$. In preparing the media, precaution must be taken to maintain the osmotic pressure of the medium series at a costant level so as not to cause any noticeable osmotic changes in cell volume. For this purpose high polymer electrolytes such as salt-free water soluble proteins (e.g. serum albumin) were successfully used. Light scattering spectra were measured for each member of the suspension series, obtaining thereby a group 
of curves similar in shape to curve (A), (B) or (C) in Fig. 3.

As in the range where $1<x<20$, cross section of light scattering, is given by equ. (3), the relative change in turbidity $\tau$ among the members of the series with varying index of refraction $\left(n_{0}\right)$ is expressed as:

$$
\begin{aligned}
\tau & =N \cdot \sigma_{s}=2 N \pi a^{2}(m-1)^{2}\left(2 \pi a / \lambda_{0}\right)^{2} \\
& =(1 / 2) N \pi^{3}\langle D\rangle^{4}(\Delta n / \lambda)^{2},
\end{aligned}
$$

where $N$ is the population density and $\lambda$ and $\lambda_{0}$ the wavelengths of the used light in vacuum and in a medium of refractive index $n_{0}$, respectively.

Consequently, if light of a proper wavelength $(\lambda)$ is so chosen as to make ratio parameter $(x)$ equal to or smaller than 20 , the square root of the turbidity $\tau$ of the suspension series desceases in proportion to $\Delta n$. We can, therefore, find the value of cellular index of refraction $n_{1}$ as an intercept on $\mathrm{x}$-axis by plotting $\sqrt{ } \tau^{\prime}$ for $n$.

Fig. 4 shows two examples of evaluation of evaluation of $n_{1}$ by the use of this linear extrapolating method. The wave lengths used were $1 \mu$ for yeast and $800 \mathrm{~m} \mu$ for chlorella, respectively. Cellular indicies of refraction obtained in this manner give, therefore, the values at the prescribed wavelengths. In contrast to the

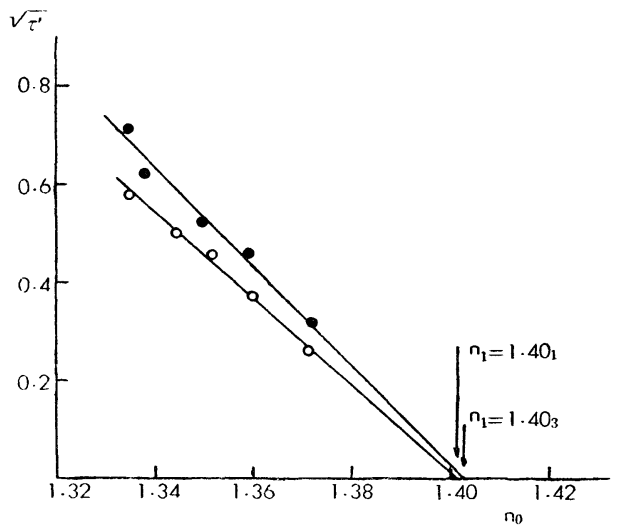

Fig. 4. Cellular indicies of refraction $\left(\mathrm{n}_{1}\right)$ as found by the extrapolating method.

Saccharomyces cerevisiae $(1 \mu)$

- Chlorella ellipsoidea $(800 \mathrm{~m} \mu)$ interprolating method which usually necessitates the use of fairly large amount of a salt-free protein as described in a previous paper ${ }^{11}$, the present one seems much more convenient in practical use.

(b) Average Cell Diameter $\langle I\rangle\rangle$ :

As numerically seen from equs. (1) and (2), the absolute value of $\tan \theta \equiv \frac{d\left[\log \left(\sigma_{s} / \pi a^{2}\right)\right]}{d\left[\log \left(1 / \lambda_{0}\right)\right]}$ decreases monotonically as cell radius increases so far as ratio prameter $x$ falls on the longer wavelength side of the spectral peak shown in Fig. 1. Average cells diameter $\langle D\rangle$ could, therefore, be obtained from the value of $|\tan \theta|$ at a properly chosen wavelength. Actually, the ratio of turbidities at two arbitrarily chosen wavelengths on an observed spectrum curve might provide a convenient measure to evaluate the value of $\langle D\rangle$.

Fig. 5 shows a calculated working curve for use in obtaining $\langle D\rangle$ from the observed values of $\tau$ at $800 \mathrm{~m} \mu$ and $665 \mathrm{~m} \mu$. The curve corresponds to 


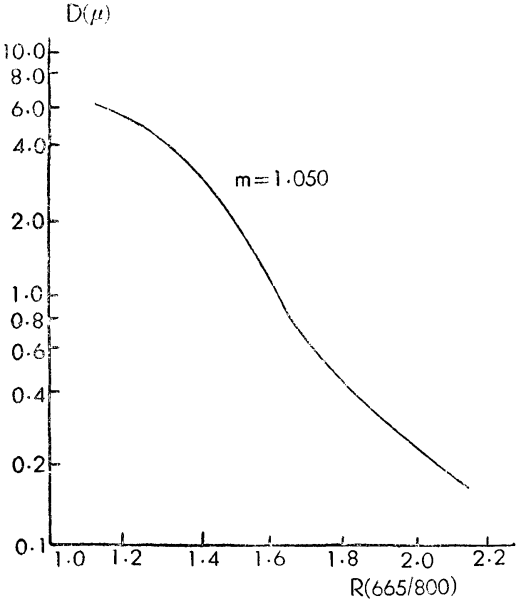

Fig. 5. Working curve for calculation of cell diameter

the case in which spherical cells of relative index of refraction $m=1.050$ are suspended in a medium of $n=1.333$. When $\langle D\rangle\langle 2 \mu$, the curve remains identical for all values of $m$, while if $\langle D\rangle \geqslant 2 \mu$, the curve takes different shape for different value of $m$ necessitating the use of a particular curve computed by using the value of $m$ observed as mentioned above.

(c) Cell Population N:

In dilute suspensions in which no multiple scattering occurs, the relation

$$
\tau=N \cdot \sigma_{s}
$$

always holds, $N$ being the cell number in unit volume. The value of $\tau$ could directly be observed and the use of $m$ and $\langle D\rangle$ obtained as described in sections (a) and (b) respectively made it possible to compute therefrom the value of $\sigma_{s}$. The population desity $(N)$ of spherical cells in suspension could thus be obtained in a perfectly spectrophotometrical manner. The values of $N$ indirectly obtained agree fairly well with those given by counting under microscope.

\section{SOME BIOLOGICAL APPLICATIONS}

In the preceding sections, basic formulae and general procedures for the analysis of light scattering spectra of microbial suspensions were described. In the present section, some examples of the application to microbiological problems will be mentioned by using Saccharomyces cerevisiae as a test microbe.

(a) Cell Volume Growth as Appeared in Spectrum of Light Scattering

In Fig. 6 are shown a group of spectral curves given by a dilute suspensions of yeast cells when aerobically grown on a Knopps medium containing yeast extract. The culture showed no indication of population increase during the observation.

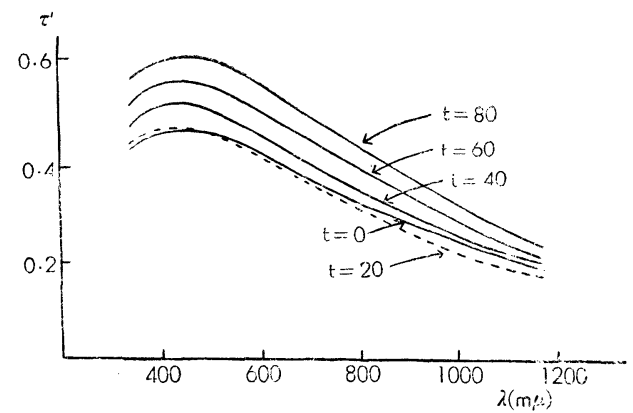

Fig. 6. Scattering spectrum change due to

As easily seen from equ. (5), volume growth (Saccharomyces cerevisiae). 
the first maximum on a turbidity-wavelength curve appears at a wavelength where $\lambda_{0}=(m-1) \pi\langle D\rangle / 2.08$. At this particular wavelength

$$
\rho \equiv 2 x(m-1)=4.16
$$

giving independently of $m$ a universal value of 3.17 as the maximum value of $\left(\sigma_{s} / \pi a^{2}\right)$. Accordingly, if population density is known, cell radius $(a)$ can be obtained from the maximum turvidity $\tau_{\max }$ by using

$$
\pi a^{2}=\tau_{\max } /(3.17 N) .
$$

As no population incraase was observed, the increase of $\tau_{\text {max }}^{\prime}$ in Fig. 6 could be ascribed to the increase of cell diameter caused by incubation. The time rate of relative increase in cell diameter is shown as curve $D$ in Fig. 7. Moreover, the peak wave-length in Fig. 6 remained practically unaltered during the growth period, indicating in accordance with equ. (6) that the cellular index of refraction $\left(n_{1}\right)$ decreased as cell diameter $\langle D\rangle$ increased. In Fig. 7 the observed change of maximum turbidity is drawn together with computed curves for the time change of $\langle D\rangle$ and $n_{1}$. In this figure $n_{1}$ represents a value of cellular index for monochromatic light of wavelength $\lambda_{\max }$ which is $420 \mathrm{~m} \mu$ in vacuum and $420 \mathrm{~m} \mu / n_{0}$ in a medium with index of refraction $n_{0}$.

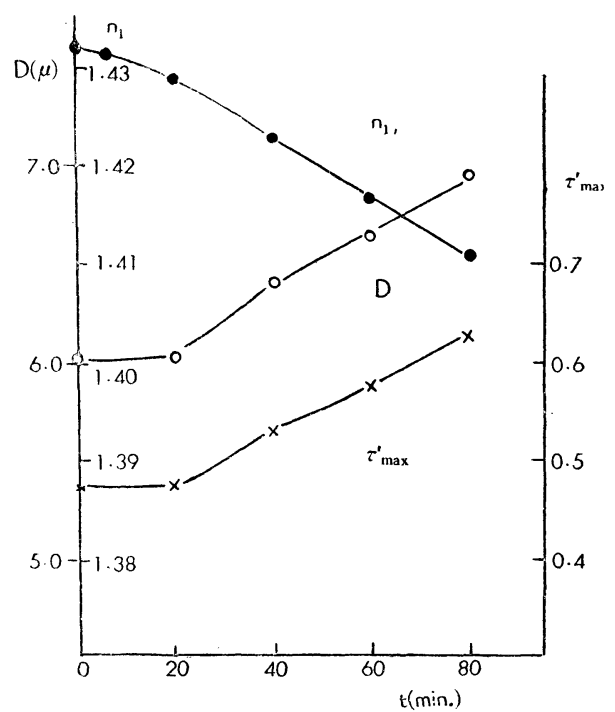

Fig. 7. Rate of change in cell parameters.

\section{(b) Osmosis as Appeared in Spectrum of Light Scattering}

If osmotic pressure difference is applied between extra-and intra-cellular media, it may possibly be balanced by the intrusion or extrusion of water passing through the semi-permeable cell membrane, causing, thereby, changes in both cell volume and cellular index of refraction. In general, turbidity cell suspension rose with the increase of cell volume and its index of refraction. If cells are suspended in a hypertonic medium, osmotic contraction occurs resulting in a decrease of cell volume and increase of refractive index, while if suspended in a hypotonic medium, osmotic expansion will be observed accompanied with an increase in cell volume and decrease in cell index of refraction. In both cases, an increase or a decrease might be observed in turbidity of the suspension, depending on which of the two opposing factors, volume effect and refractive index effect, is dominant. 
For instance, as graphically seen in cells with relative index of refraction $m=1.05$ from Fig. 8 and could exactly be computed according to equ. (4), when a cell suspension was mixed with a hypertomic solution, the turbidity would increase owing to the index effect if the cells had a relatively small diameter, say 1 or $2 \mu$, while it would decrease owing to the volume
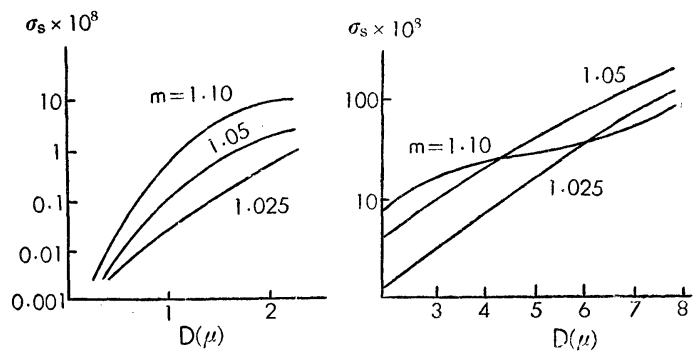

Fig. 8. Scattering cross section as functions of cell diameter (computed in accordance with equs. (1) and (2)).

effect if the cells were of a larger size, say 5 or $6 \mu$ in diameter. The suspension of cells with medium size is, therefore, expected to exhibit practically no turbidity change because of the two opposing effects mentioned

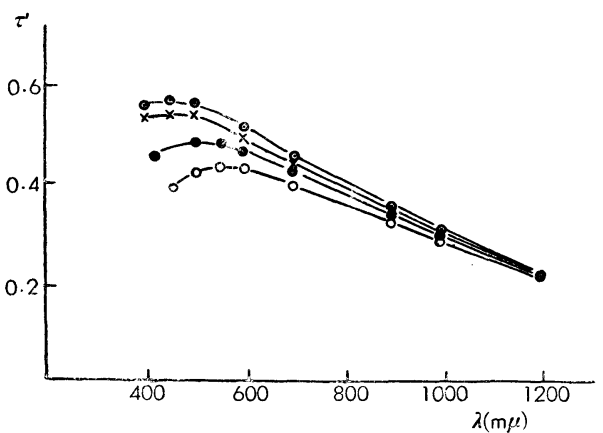

Fig. 9. Spectral changes caused by osmosis.
- $\mathrm{H}_{2} \mathrm{O}$
$\times \quad 1 \times 10^{-1} M / l(\mathrm{NaCl})$
- $5 \times 10^{-1} \mathrm{M} / \mathrm{l}(\mathrm{NaCl})$
○ $1 M / l(\mathrm{NaCl})$ above.

In Fig. 9 is shown a group of spectra of Saccharomyces cerevisiae in $\mathrm{NaCl}$ solutions of various concentrations. It appears to be due to the valume effect that the turbidity decreases in shorter wave regions with the increase of $\mathrm{NaCl}$ concentration. If so, in longer wavelength side, the turbidity change must be observed in the oppsite direction. In Fig. 10, a difference spectra of the same suspension is drawn by using a suspension with equal population in pure water as a reference. At the longest side of the observed wavelength region, the curve is of negative sign as theoretically expected. If proper organism of smaller size were used, more prominent rise of turbidity might be realized by the osmotic contraction of cells in suspension. By considering the two opposing volume and index effects in the basic formulae for turbidity of cellular suspensions, the turbidity inversion of this kind could be explained physically so far as its main features are concerned without refrerring to any particular physiological mechanism. 


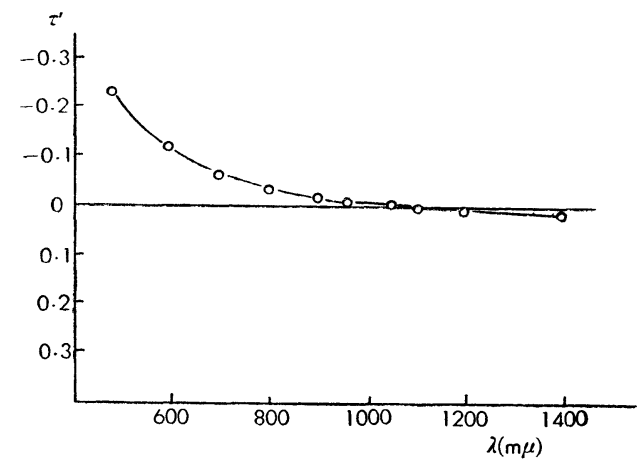

Fig. 10. Difference spectrum of yeast suspended in $\mathrm{NaCl}$ solution (referred to suspension in water).

\section{ACKNOWLEDGEMENT}

The authors are indebted to Dr. H. Iizuka of this institute for his kindness in supplying strains used in this study.

\section{SUMMARY}

By using monocellular microbes of spherical or quasi-spherical shape, spectrophotometrical studies on turbidity of cell suspension were made with the following results.

(1) Commercial spectrometers designed for solution chemistry can be used for approximate measurement of absolute turbidity of microbial suspension.

(2) Light scattering spectra of microbial suspensions could thereby be easily obtained making rossible "light scattering analysis" based on the Rayleigh-Gans' and Hart-Montroll's equations.

(3) General procedures for obtaining cellular index of refraction, cell volume and cell population were examined in the case of Saccharomyces cereuisiae and Chrorella ellipsoidea.

(4) "Light scattering analysis" gave some information on biological phenomena such as cell growth and osmosis in liquid culture.

(5) Variations of cellular index of refraction caused by volume change of cells explained the apparent inversion of turbidity change observed in osmosis of microbial suspension.

\section{REFERENCE}

(1) S. Koga and T. Fujita: J. Gen. Appl. Microbiol., 6, 101 (1960). 